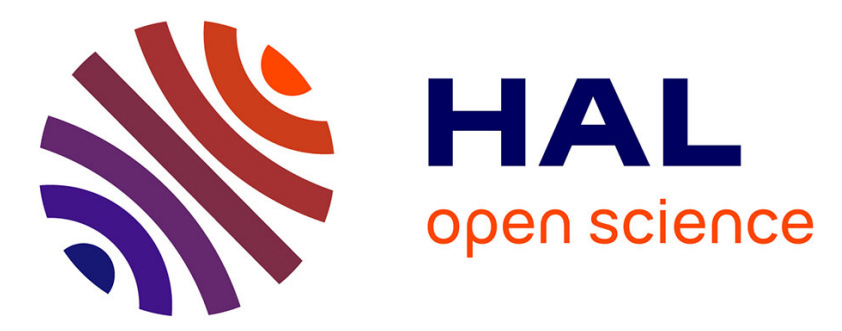

\title{
A NOTE ON EXIT TIME FOR ANCHORED ISOPERIMETRY
}

Thierry Delmotte, Clément Rau

\section{To cite this version:}

Thierry Delmotte, Clément Rau. A NOTE ON EXIT TIME FOR ANCHORED ISOPERIMETRY. Annales de la Faculté des Sciences de Toulouse. Mathématiques., 2015, 24 (4), pp.817-834. 10.5802/afst.1466 . hal-01963536

\section{HAL Id: hal-01963536 https://hal.science/hal-01963536}

Submitted on 21 Dec 2018

HAL is a multi-disciplinary open access archive for the deposit and dissemination of scientific research documents, whether they are published or not. The documents may come from teaching and research institutions in France or abroad, or from public or private research centers.
L'archive ouverte pluridisciplinaire HAL, est destinée au dépôt et à la diffusion de documents scientifiques de niveau recherche, publiés ou non, émanant des établissements d'enseignement et de recherche français ou étrangers, des laboratoires publics ou privés. 


\title{
A NOTE ON EXIT TIME FOR ANCHORED ISOPERIMETRY
}

\author{
THIERRY DELMOTTE, CLÉMENT RAU
}

\begin{abstract}
Let $\left(X_{n}\right)_{n \geq 0}$ be a reversible random walk on a graph $G$ satisfying an anchored isoperimetric inequality. We give upper bounds for exit time (and occupation time in transient case) by $\mathrm{X}$ of any set which contains the root. This article covers many results of [11].

Résumé

Soit $\left(X_{n}\right)_{n \geq 0}$ une marche aléatoire réversible sur un graphe $G$ vérifiant une inégalité isopérimétrique ancrée. Nous obtenons une majoration du temps de sortie de tout ensemble connexe contenant un point ancre (et du temps de passage dans le cas transient) de la marche X. Cet article reprend un grand nombre de résultats de [11].
\end{abstract}

\section{Contents}

1. Introduction

1.1. Overview about rooted isoperimetric inequality.

1.2. Continuous space setting.

1.3. Results in discrete setting

2. Green functions properties and exit time.

2.1. Definitions and harmonicity.

2.2. Differential inequation for the size of Green functions level sets.

2.3. Upper bound for exit time, proof of Theorem 1.4

2.4. Examples of $\mathcal{F}$ functions.

3. Applications

3.1. Transience

3.2. Speed

3.3. Random environment on $\mathbb{Z}^{d}$, including supercritical percolation

References

\section{INTRODUCTION}

Among many connections linking the geometry of a graph and the behaviour of its simple random walk, one important tool is isoperimetry. A graph $G$ satisfies the (uniform) isoperimetric inequality if there exists a constant $C>0$ such that for any finite set $A$, we have:

$$
\frac{|\partial A|}{\mathcal{F}(|A|)} \geq C
$$

where $\partial A$ is the set $\{(x, y)$ edge of $G$, such that $x \in A$ and $y \notin A\},|B|$ stands for the cardinal of $B$ and $\mathcal{F}$ is a non decreasing positive function. 
Already present in the celebrated work of Nash [20], the idea was made clear since the seminal work of Varopoulos [26]. A discrete version for graphs is in [9]. The isoperimetry conditions are various, geometric or functional. For instance the inequality above yields a $L^{2}$ type of Faber-Krahn inequality proposed by Grigor'yan in [14], and Coulhon has shown in [8] that it gives an upper bound of the iterated transition probabilities of the random walk.

The problem with uniform isoperimetric inequality is its unstability under random perturbations like percolation. For example, in the super-critical percolation of $\mathbb{Z}^{d}(d \geq 2)$, if $G$ is the infinite cluster assuming it contains the origin, you can find some linear piece as long as you want, if you go far enough from the origin. And then classical isoperimetry inequality fails, although it holds in $\mathbb{Z}^{d}$. If one studies the "ant in the labyrinth" of de Gennes [10], one needs a weaker version of isoperimetry which can be robust, as introduced in the two last decades by Thomassen in [25] and next by Benjamini, Lyons and Schramm in [4]. It is called anchored or rooted isoperimetric inequality. Here is the definition.

For a graph $G$, we denote $V(G)$ the set of vertices and $E(G)$ the set of edges.

Definition 1.1. Let $\mathcal{F}$ a positive increasing function defined on $\mathbb{R}_{+}$. Let $G$ a graph and $o \in G$. We say that $G$ satisfies an anchored (or rooted) $\mathcal{F}$-isoperimetric inequality at $o$ if there exists a constant $C_{\mathrm{IS}}>0$ such that for any connected set $A$ which contains o we have:

$$
\frac{|\partial A|}{\mathcal{F}(|A|)} \geq C_{\mathrm{IS}}
$$

$\partial A$ is the set $\{(x, y) \in E(G) ; x \in A$ and $y \notin A\}$.

Under this anchored isoperimetric inequality, our main result is Theorem 1.4 with upper bounds for exit and local times.

The introduction of this kind of inequalities can be justified as follows. In order to overlook the isoperimetric holes that may appear, you restrict only on connected sets that contain a fixed point. The effect would be a kind of average that makes these holes negligible. This is precisely what we expect for super-critical percolation.

When $\mathcal{F}(x)=x^{1-1 / d}$, we will say that $G$ satisfies a $d$-dimensional isoperimetric inequality. When $\mathcal{F}=i d$ and $G$ has bounded degree there is an equivalent version of this definition which reads as follows:

$G$ satisfies a strong anchored (or rooted) isoperimetric inequality if

$$
\lim _{n \rightarrow \infty} \inf \left\{\frac{|\partial S|}{|S|} ; S \text { connected, } v \in S \text { and }|S| \geq n\right\}:=i(G)
$$

is positive (non zero).

This definition does not depend on the choice of the fixed vertex whereas in the previous definition, the constant $C_{\text {IS }}$ depends on the point o.

Our object here is to examine what anchored isoperimetric inequality implies for random walk. Our hope is that it could be useful for instance in the still very open problem about spectral dimension of the critical incipient infinite cluster (IIC) which could not be $4 / 3$ in low dimensions as stated by the Alexander-Orbach conjecture [1]. See the lecture of Barlow [3] for an introduction to these questions. Note that it should not be useful in high dimension. A recent discussion with Gady Kozma tells us that there is no hope to prove an anchored isoperimetric inequality. The main reason is the existence of a backbone in the IIC (see [16]). The backbone 
of the IIC is analogous to the unique infinite line of descent. Roughly speaking, the IIC can be seen as as a single path embedded into $\mathbb{Z}^{d}$ (the backbone) with some finite trees attached at some points of the backbone. This picture shows you that no (anchored) isoperimetric inequality may be satisfied. For low dimension, the question stays open and isoperimetry is perhaps a relevant tool.

\subsection{Overview about rooted isoperimetric inequality.}

The first result known for rooted $\mathcal{F}$-isoperimetric inequality is due to Thomassen. In [25], it is proved that the simple random walk on a graph $G$ is transient if $G$ satisfies a rooted $\mathcal{F}$-isoperimetric inequality such that $\sum_{k} \mathcal{F}(k)^{-2}<\infty$. The main step of the proof is to extract a subdivision of the dyadic tree from the initial graph. Then, thanks to hypothesis, it is possible to construct a finite flow on the tree, which proves that the tree is transient.

It was long afterwards that other results did appear for anchored isoperimetry. In 2000 Virag has studied the case of strong anchored isoperimetric inequality. In [27], it is proved that strong anchored isoperimetric inequality on graphs with bounded geometry, implies a positive lim inf speed. Moreover Virag proves that in this case, transitions probabilities at time $n$ of the random walk are bounded by $e^{-n^{1 / 3}}$.

Later, still when $\mathcal{F}=i d$, Chen and Peres have proved that if $G$ satisfies a strong anchored isoperimetric inequality then so does every infinite cluster of independant percolation with parameter $p$ sufficiently closed to 1 . Next, they have shown that strong anchored isoperimetry is preserved under a random stretch if, and only if, the stretching law has an exponential tail. They also proved that for a supercritical Galton Watson tree $\mathbb{T}$ given nonextinction, we have $i(\mathbb{T})>0$ a.s.

There is an important collection of conjectures relating to anchored expansion. One of them is to know if anchored isoperimetric inequality is a good tool to prove an invariance principle in random environment of $\mathbb{Z}^{d}$. Another challenge is to understand if a general anchored isoperimetric inequality may imply an upper bound of $p_{n}(x, y)$. From uniform isoperimetric inequality, the standard proof is to show that a Nash inequality holds for all finitely supported functions $f$ defined on graph $G$ (see [21] for instance). For this aim, we apply isoperimetric inequality to the level sets $\{f \geq t\}$. To get only upper bounds for the transition kernel, a careful reading of the proof, shows you that it is sufficient to prove Nash inequality restricted to functions $f$ of the form $f()=.\left(P^{n} 1_{\{a\}}\right)($.$) , where a$ belongs to $G$ and $n$ is an integer (to avoid parity problems, you can work with the lazy random walk that stays where it is with probability $1 / 2$, and jumps uniformly on one his neighbors otherway). Now, assume that only a rooted isoperimetric inequality at the origin $o$ holds on $G$. One may hope that it implies upper bounds for $p^{n}(o, o)$. To have the proof work, you see that the level sets of $P^{n} 1_{\{o\}}$ must be connected and must contain the origin $o$. It turns out that this fact is wrong in general, as you can easily check by drawing some graph. As $n$ grows, when the unit mass at the origin $o$ spreads out on the graph, $P^{n} 1_{\{o\}}$ may accumulate in some part of it and become bigger than at the origin.

In a recent work of Morris and Peres [19], they introduce a new probabilistic technique linking isoperimetry and transition kernel, but the same problem occurs.

1.2. Continuous space setting. The paper is written in the discrete space setting of graphs. The reason is that anchored isoperimetric inequality is a natural tool 
in random media and is therefore more associated with this setting. In fact the continuous setting (of Riemannian manifolds for instance) works as well, and may be, the proofs are far more readable. As both an introduction to our technique and an illustration of what the continuous setting results would look like, we begin with a key result written in this setting. Details, especially from potential theory, will only appear later in the paper for graphs.

Let $M$ be a Riemannian manifold with an anchored isoperimetric inequality at root $o$, that is (1) for finite volume smooth connected domains $A$ containing $o$. Precisely, $|A|=m(A)$ for the Riemannian volume element $m$ and $|\partial A|=\mu(\partial A)$ for the Riemannian volume element $\mu$ on the smooth submanifold $\partial A$.

Now let fix some $A$ and consider the Brownian motion on $M$ starting at $o$ and killed when hitting $\partial A$ at time $\tau_{A}$. We denote $p_{t}^{A}$ its submarkovian kernel, $A_{s}$ the level sets of Green function and $u(s)$ their measures.

$$
A_{s}=\left\{x \in A, G^{A}(x)=\int_{0}^{\infty} p_{t}^{A}(x) \mathrm{d} t \geq s\right\}, \quad u(s)=m\left(A_{s}\right) .
$$

Thanks to harmonic properties of $G^{A}$, these level sets are connected and contain the root. Thus, they will also satisfy (1). In the following we use $\mu$ for any $s$ and also $\nu$ denoting the inward unit normal vector field on $\partial A_{s}$. The inward direction is chosen to have $G^{A}$ increasing.

Theorem 1.2. The anchored isoperimetric inequality yields a differential inequation

$$
u^{\prime}(s) \leq-\left(C_{\mathrm{IS}} \mathcal{F}(u(s))\right)^{2}
$$

This naturally leads to upper estimates of $u(s)$ and $\mathbb{E}\left(\tau_{A}\right)=\int_{0}^{\infty} u(s) \mathrm{d}$.

For instance if $\mathcal{F}(u)=u^{1-1 / d}, \mathbb{E}\left(\tau_{A}\right) \leq C m(A)^{2 / d}$, and if $\mathcal{F}(u)=u, \mathbb{E}\left(\tau_{A}\right) \leq C \ln m(A)$.

Proof. Schwarz inequality

$$
\left(C_{\mathrm{IS}} \mathcal{F}(u(s))\right)^{2} \leq \mu\left(\partial A_{s}\right)^{2}=\left(\int_{\partial A_{s}} \mathrm{~d} \mu\right)^{2} \leq \int_{\partial A_{s}} \frac{\partial G^{A}}{\partial \nu} \mathrm{d} \mu \int_{\partial A_{s}} \frac{\mathrm{d} \mu}{\partial G^{A} / \partial \nu}
$$

involves the flow

$$
\int_{\partial A_{s}} \frac{\partial G^{A}}{\partial \nu} \mathrm{d} \mu=1
$$

and the derivative of $u$ since whith the co-area formula,

$$
u(s)=\int_{G^{A} \geq s} \mathrm{~d} m=\int_{s}^{\infty}\left(\int_{G^{A}=t} \frac{\mathrm{d} \mu}{\partial G^{A} / \partial \nu}\right) \mathrm{d} t
$$

This yields the differential inequation.

For $\mathcal{F}(u)=u^{1-1 / d}$, computations may be avoided if we compare with the case when $A$ is a ball of radius $R$ in $\mathbb{R}^{d}$. Then $\partial G^{A} / \partial \nu$ is constant, all inequalities are equalities and the result should be that $\mathbb{E}\left(\tau_{A}\right)$ is like $R^{2}$.

Application of this Schwarz inequality is already apparent in [13], [23] or [17] to establish a recurrence criterion or estimate resistance. 


\subsection{Results in discrete setting.}

Let $G$ be a graph and $o$ one particular vertex. Consider a random walk $\left(X_{n}\right)_{n \geq 0}$ on $G$ with transition probability $p(.,$.$) and assume there exists a reversible measure$ $m$ for $X$. We use the symmetric kernel $\mu(x, y):=m(x) p(x, y)$ to measure surfaces:

$$
\forall A \subset G, \quad \mu(\partial A)=\sum_{x \in A, y \notin A} \mu(x, y) .
$$

In this setting the anchored isoperimetric inequality reads:

Definition 1.3. We say $G$ satisfies the anchored isoperimetric inequality at root o with increasing function $\mathcal{F}$ when for any connected $o \in A \subset G$,

$$
\frac{\mu(\partial A)}{\mathcal{F}(m(A))} \geq C_{I S}
$$

"Connected" means that one can find a discrete path in A between any two points for which $p\left(x_{i}, x_{i+1}\right)$ is positive when $x_{i}, x_{i+1}$ are following points.

No distance will play a role here and the graph is not assumed to be locally finite.

We denote $\mathbb{P}_{x}$ [resp $\mathbb{E}_{x}$ ] the law of the walk starting from point $x$ [resp the expectation], $\tau_{A}$ the exit time and $l_{A}$ the occupation time (which may be infinite if $X$ is not transient):

$$
\tau_{A}=\inf \left\{k \geq 0 ; X_{k} \notin A\right\}, \quad l_{A}=\operatorname{card}\left\{k \in \mathbb{N} ; X_{k} \in A\right\} .
$$

Theorem 1.4. If $G$ satisfies (2), then for any subset $A$ we have:

$$
\mathbb{E}_{o}\left(\tau_{A}\right) \leq 2 \int_{0}^{\infty} v_{+}^{A}(s) \mathrm{d} s
$$

$$
\text { and } \quad \mathbb{E}_{o}\left(l_{A}\right) \leq 2 \int_{0}^{\infty} v_{+A}(s) \mathrm{d} s,
$$

where $v^{A}, v$ are solutions of $\left\{\begin{array}{l}v^{A}(0)=m(A) \\ \left(v^{A}\right)^{\prime}=-\left(C_{I S} \mathcal{F}\left(v^{A}\right)\right)^{2},\end{array} \quad\right.$ and $\left\{\begin{array}{l}v(0)=+\infty \\ v^{\prime}=-\left(C_{I S} \mathcal{F}(v)\right)^{2} .\end{array}\right.$

The truncations in indices mean

$v_{+}^{A}(s)=\left\{\begin{array}{l}0 \text { if } v^{A}(s) \leq 0 \\ v^{A}(s) \text { otherwise. }\end{array} \quad\right.$ and $v_{+A}(s)=\left\{\begin{array}{l}0 \text { if } v(s) \leq 0 \\ m(A) \text { if } v(s) \geq m(A) \\ v(s) \text { otherwise. }\end{array}\right.$

For comparison when $X$ is transient, note that

$$
\int_{0}^{\infty} v_{+}^{A}(s) \mathrm{d} s=\int_{v^{-1}(m(A))}^{\infty} v_{+}(s) \mathrm{d} s .
$$

We consider usual functions $\mathcal{F}$ in Section 2.4. It is sometimes useful to precise the values $\mathcal{F}(x)=\mathcal{F}(m(o))$ for $x \leq m(o)$, which is justified in Proposition 2.4.

The paper is constructed as follows: in section 2, we establish a few properties of Green functions, that allow us to apply anchored isoperimetric inequality for its levels sets. This gives us a differential inequation for the size of the level sets. We then deduce an upper bound for exit time. In the last section, we give some applications of Theorem 1.4. We firt retrieve some results of Virag and Thomassen. Then, we investigate random environments (and super-critical percolation). 


\section{Green Functions properties And EXit time.}

2.1. Definitions and harmonicity. The submarkovian kernel of the killed random walk is $p^{A}(x, y)= \begin{cases}p(x, y) & \text { if } x \in A, \\ 0 & \text { otherwise. }\end{cases}$

Although Theorem 1.4 is true for $A$ non connected, we have in this section to assume $A$ is connected. When $X$ is transient, Green function may be defined for the non-killed random walk and we can consider $A=G$ (or the connected component of $o$ if $G$ was not connected, which would have little interest). This leads to the result for $l_{A}$ in next section.

The discrete Laplacian is

$$
\triangle^{A} f=\left(I d-P^{A}\right) f
$$

where $P^{A}$ is the operator defined on functions which are zero outside $A$ by

$$
\begin{aligned}
P^{A} f(x) & =\mathbb{E}_{x}\left(f\left(X_{1}\right) 1_{\left\{x, X_{1} \in A\right\}}\right) \\
& =\sum_{y \in A} p^{A}(x, y) f(y) .
\end{aligned}
$$

The Green function is

$$
G^{A}(x, y)=\frac{1}{m(y)} \sum_{k \geq 0} \mathbb{P}_{x}^{A}\left(X_{k}=y\right) .
$$

In particular we denote $G^{A}(x)=G^{A}(o, x)$. Note that $G^{A}(x)=0$ if $x \notin A$.

Recall that reversibility means $p(x, y) / m(y)=p(y, x) / m(x)$. In other words $p(x, y) / m(y)$ is the precise analog of a density kernel in $y$ starting from $x$ and is symmetric. This explains the factor $1 / m(y)$ in the definition of $G^{A}$ which is symmetric for $x, y \in A$.

Proposition 2.1. $\triangle \triangle^{A} G^{A}=\frac{\delta_{0}}{m(0)}$

Proof. For all $x \in A$ we have :

$$
\begin{aligned}
\triangle^{A} G^{A}(x) & =\left[\left(I d-P^{A}\right)\left(G^{A}\right)\right](x) \\
& =\frac{1}{m(x)} \sum_{k \geq 0} \mathbb{P}_{o}^{A}\left(X_{k}=x\right)-\sum_{k \geq 0} \sum_{y \in A} \frac{p^{A}(x, y)}{m(y)} \mathbb{P}_{o}^{A}\left(X_{k}=y\right) \\
& =\frac{1}{m(x)} \sum_{k \geq 0} \mathbb{P}_{o}^{A}\left(X_{k}=x\right)-\sum_{k \geq 0} \sum_{y \in A} \frac{p^{A}(y, x)}{m(x)} \mathbb{P}_{o}^{A}\left(X_{k}=y\right) \\
& =\frac{1}{m(x)} \sum_{k \geq 0} \mathbb{P}_{o}^{A}\left(X_{k}=x\right)-\sum_{k \geq 0} \frac{1}{m(x)} \mathbb{P}_{o}^{A}\left(X_{k+1}=x\right) \\
& =\frac{\mathbb{P}_{o}^{A}\left(X_{0}=x\right)}{m(x)} \\
& =\frac{\delta_{0}(x)}{m(0)}
\end{aligned}
$$

And for $x \notin A$, we have $\triangle^{A} G^{A}(x)=0$.

Corollary 2.2. $G^{A}$ is harmonic on $A \backslash o$. As a consequence the level sets $A_{s}=\left\{x \in A ; G^{A}(x) \geq s\right\}$ are connected and contain o. Moreover the inward flow 
through any $\partial A_{s}$ is 1 or more generally for any $B \subset A$ :

$$
\sum_{(x, y) \in \partial B} \mu(x, y) \nabla_{(y, x)} G^{A}=1_{\{o \in B\}} .
$$

The surface notations are $\partial B=\{(x, y) ; x \in B, y \notin B\}$ and $\nabla_{(y, x)} f=f(x)-$ $f(y)$.

Proof. For all $x \in A$, Propostion 2.1 may be written

$$
\sum_{y \in G} p^{A}(x, y)\left(G^{A}(x)-G^{A}(y)\right)=\frac{\delta_{0}(x)}{m(0)} .
$$

Summing over $x$ in $B$ with respect to $m$ we get

$$
\sum_{x \in B} \sum_{y \in G} m(x) p^{A}(x, y)\left(G^{A}(x)-G^{A}(y)\right)=1_{\{o \in B\}} .
$$

Now the usual integration by parts becomes in this discrete summation a cancellation of terms by symmetry when $y$ also belongs to $B$. Only (5) remains.

Maximum principle and properties of level sets $A_{s}$ may be extracted from this result when $o \notin B$. In this case the flow is 0 so there must be an edge $x, y$ with $G^{A}(y) \geq G^{A}(x)$. This leads to a contradiction if there was a connected component of $A_{s}$ not containing $o$.

2.2. Differential inequation for the size of Green functions level sets. We use a linearized version of $m\left(A_{s}\right)$, namely

$$
u(s)=\sum_{x \in A_{s}, y \in G} \mu(x, y) \frac{G^{A}(x)-\max \left\{s, G^{A}(y)\right\}}{G^{A}(x)-G^{A}(y)} .
$$

For $x \in A_{s}$ such that $\mu(x, y)>0 \Rightarrow y \in A_{s}$, the contribution of $x$ is indeed $m(x)$. Furthermore $u(s) \leq m\left(A_{s}\right)$. The reason for this definition is to have:

Lemma 2.3. Piecewise linear function u has left derivative

$$
u^{\prime}(s)=-\sum_{(x, y) \in \partial A_{s}} \frac{\mu(x, y)}{\nabla_{(y, x)} G^{A}} .
$$

Proof. Variation in $s$ in the definition of $u(s)$ comes from the $y$ 's such that $G^{A}(y)<$ $s$, that is $y \notin A_{s}$. This is clear but note that it uses $G^{A} \equiv 0$ outside $A$ and this would not be correct for small values of $s$ and the $\tilde{u}$ at page 9 when occupation time is considered.

Proposition 2.4. If $G$ satisfies (2), then:

$$
u^{\prime} \leq-\left(C_{\mathrm{IS}} \mathcal{F}(u)\right)^{2} .
$$

Proof. Same Schwarz inequality as for Theorem 1.2:

$$
\begin{aligned}
\left(C_{\mathrm{IS}} \mathcal{F}(u(s))\right)^{2} & \leq\left(C_{\mathrm{IS}} \mathcal{F}\left(m\left(A_{s}\right)\right)\right)^{2} \\
& \leq \mu\left(\partial A_{s}\right)^{2} \\
& \leq\left(\sum_{(x, y) \in A_{s}} \mu(x, y) \nabla_{(y, x)} G^{A}\right)\left(\sum_{(x, y) \in \partial A_{s}} \frac{\mu(x, y)}{\nabla_{(y, x)} G^{A}}\right) \\
& =-u^{\prime}(s) .
\end{aligned}
$$


This is of course correct when $u>0$, that is when $A_{s}$ is not empty and contains $o$. It works therefore with $\mathcal{F}(x)=\mathcal{F}(m(o))$ for $x \leq m(o)$.

2.3. Upper bound for exit time, proof of Theorem 1.4. Exit time (or occupation time in transient case) is linked from Green functions by the following lemma.

Lemma 2.5. For any set $A$ we have:

(i) $\mathbb{E}_{o}\left(\tau_{A}\right)=\sum_{x \in A} m(x) G^{A}(x)$,

(ii) $\mathbb{E}_{o}\left(l_{A}\right)=\sum_{x \in A} m(x) G(x)$ in the transient case.

Proof. Given a path $\gamma=\left(\gamma_{0}, \gamma_{1}, \ldots, \gamma_{n}\right)$ from $\gamma_{0}=o$ to $A^{c}$, that is only $\gamma_{n} \notin A$, we denote its probability $\mathbb{P}(\gamma)=p\left(\gamma_{0}, \gamma_{1}\right) \ldots p\left(\gamma_{n-1}, \gamma_{n}\right)$. Its length $l(\gamma)=n=$ $\sum_{x \in A} N_{x}(\gamma)$ where $N_{x}(\gamma)$ is the number of indices $i$ such that $\gamma_{i}=x$. This yields (i) since

$$
\mathbb{E}_{o}\left(\tau_{A}\right)=\sum_{\gamma} l(\gamma) \mathbb{P}(\gamma) \quad \text { and } \quad G^{A}(x)=\frac{1}{m(x)} \sum_{\gamma} N_{x}(\gamma) \mathbb{P}(\gamma)
$$

We adapt this argument to prove (ii). We keep $\gamma_{n} \notin A$ and $\gamma_{n-1} \in A$ but we may have $\gamma_{i} \notin A$ for $i<n-1$. The probability of the path is not easy to compute but denotes

$$
\mathbb{P}(\gamma)=\mathbb{P}_{0}\left(\forall i \leq n, X_{i}=\gamma_{i} \text { and } \forall i \geq n, X_{i} \notin A\right)
$$

We also replace the length $l(\gamma)$ by the natural occupation time $N_{A}(\gamma)$.

Now we could use $\sum_{x \in A} m(x) G^{A}(x)=\int_{0}^{\infty} m\left(A_{s}\right) \mathrm{d} s$. It is a little more intricate since we have control on $u$ which is a linearized version of $m\left(A_{s}\right)$.

Lemma 2.6. For any set $A$ we have:

$$
\int_{0}^{\infty} u(s) \mathrm{d} s=\sum_{x \in A, y \in G} \mu(x, y) \min \left\{G^{A}(x), \frac{G^{A}(x)+G^{A}(y)}{2}\right\} .
$$

Proof. From the definition of $u$ we just have to compute carefully

$$
\int_{0}^{\infty} \frac{G^{A}(x)-\max \left\{s, G^{A}(y)\right\}}{G^{A}(x)-G^{A}(y)} 1_{x \in A_{s}} \mathrm{~d} s .
$$

We now have completed the proof of (3) in Theorem 1.4. Factor 2 in the righthand sides comes from

$$
\begin{aligned}
\sum_{x \in A} m(x) G^{A}(x) & =\sum_{x \in A, y \in G} \mu(x, y) G^{A}(x) \\
& \leq 2 \sum_{x \in A, y \in G} \mu(x, y) \min \left\{G^{A}(x), \frac{G^{A}(x)+G^{A}(y)}{2}\right\} .
\end{aligned}
$$

As far as (3) is concerned, the result first for $A$ connected is clearly sufficient.

To prove (4), we first use the differential inequation with $A=G$, that is we obtain $u(s) \leq v(s)$ for

$$
u(s)=\sum_{G(x) \geq s, y \in G} \mu(x, y) \frac{G(x)-\max \{s, G(y)\}}{G(x)-G(y)} .
$$


Then we argue (here $A$ is not necessarly connected)

$$
\mathbb{E}_{o}\left(l_{A}\right) \leq 2 \sum_{x \in A, y \in G} \mu(x, y) \min \left\{G(x), \frac{G(x)+G(y)}{2}\right\} \leq 2 \int_{0}^{\infty} \tilde{u}(s) \mathrm{d} s,
$$

where

$$
\tilde{u}(s)=\sum_{x \in A_{s}, y \in G} \mu(x, y) \frac{G(x)-\max \{s, G(y)\}}{G(x)-G(y)} .
$$

It is clear that $\tilde{u}(s) \leq u(s) \leq v(s)$ and $\tilde{u}(s) \leq m(A)$. That ends up proof of Theorem 1.4.

2.4. Examples of $\mathcal{F}$ functions. As a complement to Theorem 1.4, we give upper bounds for exit time for classical functions $\mathcal{F}$. If $\mathcal{F}(x)=x^{1-1 / d}$ as in $\mathbb{Z}^{d}$ then Theorem 1.4 gives

$$
\begin{aligned}
\mathbb{E}\left(\tau_{A}\right) & \leq \frac{d}{C_{\mathrm{IS}}^{2}} m(A)^{2 / d} \\
\text { and } \mathbb{E}\left(l_{A}\right) & \leq \frac{d^{2}}{C_{\mathrm{IS}}^{2}(d-2)} m(A)^{2 / d} \text { for } d>2 .
\end{aligned}
$$

Indeed for $d>2$ the Thomassen criterium implies the transience, see below. The computations involve

$$
\begin{aligned}
v^{A}(s) & =\left(m(A)^{\frac{2-d}{d}}-C_{\mathrm{IS}}^{2} \frac{2-d}{d} s\right)^{\frac{d}{2-d}} \text { for } d \neq 2, \\
v(s) & =\left(C_{\mathrm{IS}}^{2} \frac{d-2}{d} s\right)^{\frac{-d}{d-2}} \text { for } d>2 \\
\text { and } v^{A}(s) & =m(A) e^{-C_{\mathrm{IS}}^{2} s} \text { for } d=2 .
\end{aligned}
$$

If $\mathcal{F}(x)=x$ as in a non-amenable graph then Theorem 1.4 gives

$$
\begin{aligned}
\mathbb{E}\left(\tau_{A}\right) & \leq \frac{1}{C_{\mathrm{IS}}^{2}}\left(1+2 \ln \frac{m(A)}{m(o)}\right) \\
\text { and } \mathbb{E}\left(l_{A}\right) & \leq \frac{1}{C_{\mathrm{IS}}^{2}}\left(3+2 \ln \frac{m(A)}{m(o)}\right) .
\end{aligned}
$$

Here we need the precision $\mathcal{F}(x)=m(o)$ for $x \leq m(o)$ so that

$$
\frac{1}{v^{A}(s)}=\frac{1}{m(A)}+C_{\mathrm{IS}}^{2} s
$$

does not arise any issue of integration for $s \rightarrow \infty$.

We can summarize these computations in:

Proposition 2.7. Let $G$ a graph satisfying a weighted anchored isoperimetric inequality with function $\mathcal{F}$ and anchored expansion constant $C_{I S}$ (see (2)). Then, there exists constants $c(d)$ and $c$ such that:

- if $\mathcal{F}(x)=x^{1-\frac{1}{d}}(d \geq 3)$ we have: $\mathbb{E}_{o}\left(l_{A}\right) \leq c(d) m(A)^{\frac{2}{d}}$,

- if $\mathcal{F}(x)=x^{\frac{1}{2}}(d=2)$ we have: $\mathbb{E}_{o}\left(\tau_{A}\right) \leq c(d) m(A)$,

- if $\mathcal{F}(x)=x$ we have: $\mathbb{E}_{o}\left(\tau_{A}\right) \leq \mathbb{E}_{o}\left(l_{A}\right) \leq c \ln (m(A))$. 
Remark 2.8. These inequalities are sharp. Take the particular case where G satisfies a not anchored isoperimetric inequality.

Remark 2.9. Notice that the constant $c(d)$ is proportional to $1 / C_{I S}^{2}$. There exists a constant $c_{1}(d)>0$ such that:

$$
c(d)=\frac{c_{1}(d)}{C_{I S}^{2}}
$$

\section{Applications}

3.1. Transience. We retrieve Thomassen result's cited in the introduction. Indeed, proposition 2.4 provides a new proof of the transience of the random walk under the summability assumption on $\mathcal{F}$ without introducing the complex construction of dyadic subtrees by Thomassen. Assume

$$
\int_{1}^{+\infty} \frac{1}{\mathcal{F}(n)^{2}}<+\infty
$$

for $\mathcal{F}: \mathbb{R}_{+} \rightarrow \mathbb{R}_{+}^{*}$, not decreasing, with $\mathcal{F}(0)=0$ and let us prove transience with the help of proposition 2.4 .

Let $A$ a connected subset of $G$ containing the origin and consider random walk killed whenever it leaves $A$ and the associated Green function $G_{A}$. Integrating the differential inequation between time 0 and $\mathrm{t}$ yields:

$$
\int_{u(t)}^{u(0)} \frac{d s}{\mathcal{F}(s)^{2}} \geq C_{I S}^{2} t
$$

$\int_{1}^{u(0)} \frac{d s}{\mathcal{F}(s)^{2}}$ is bounded by a constant independant of $A$. Indeed, thanks to hypothesis (6), for all subset $A$ we have: $\int_{1}^{u(0)} \frac{d s}{\mathcal{F}(s)^{2}}=\int_{1}^{m(A)} \frac{1}{\mathcal{F}(s)^{2}} d s \leq \int_{1}^{+\infty} \frac{d s}{\mathcal{F}(s)^{2}}<+\infty$. So for large enough t which depends only on $C_{I S}$ and $\mathcal{F}$, inequality (7) turns into:

$$
\int_{u(t)}^{1} \frac{d s}{\mathcal{F}(s)^{2}} \geq \frac{1}{2} C_{I S}^{2} t .
$$

Then, we deduce that:

$$
\lim _{t \rightarrow+\infty} u(t)=0 \text { uniformly in } A .
$$

In particular, there exists $t_{0}$ independant of $A$ such that for all $t \geq t_{0}, u(t)<$ $\inf _{G} m$. Therefore by definition of $u$ we get that for all set $A, G_{A} \leq t_{0}$. Now we can make $A$ growing and finally we deduce that $G<+\infty$ so the walk is transient.

3.2. Speed. When $\mathcal{F}=i d$, the upper bound of the exit time gives us that the speed of the random walk is positive. We retrieve a weak version of Virag's result. We assume in this subsection that the graph has uniformly localy bounded valency. Let $d(a, b)$ denote the graph distance between point $a$ and $b$.

Proposition 3.1. Let $G$ be a graph satisfying (1) with $\mathcal{F}=i d$ and let $\left(X_{n}\right)_{n}$ be a simple random walk on $G$. Then we have:

$$
\mathbb{P}\left(\lim _{n} \frac{d\left(o, X_{n}\right)}{n}=0\right)=0 .
$$


Proof. Assume there exists $\epsilon>0$ such that $\mathbb{P}\left(\lim _{n} \frac{d\left(o, X_{n}\right)}{n}=0\right)>\epsilon$. So, we have:

$$
\forall \alpha>0 \quad \mathbb{P}\left(\exists N_{\alpha} \forall n \geq N_{\alpha} \quad \frac{d\left(o, X_{n}\right)}{n} \leq \alpha\right)>\epsilon
$$

By considering the event $E_{q}=\left\{\exists N_{\alpha}<q, \forall n \geq N_{\alpha} \quad \frac{d\left(o, X_{n}\right)}{n} \leq \alpha\right\}$ and by continuity of measure $\mathbb{P}$, we get:

$$
\exists N_{\alpha} \geq 0 \quad \mathbb{P}\left(\forall n \geq N_{\alpha} \quad \frac{d\left(o, X_{n}\right)}{n} \leq \alpha\right)>\frac{\epsilon}{2} .
$$

Take now $R>0$, we have:

$$
\mathbb{P}\left(\forall n \in\left[N_{\alpha} ; \frac{R}{\alpha}\right] \quad d\left(o, X_{n}\right)<\alpha n\right)>\frac{\epsilon}{2} .
$$

On this event we have: $l_{B(o, R)} \geq \frac{R}{\alpha}-N_{\alpha}$, where $l_{A}$ is the local time of $X$ in the set $A$, which is well defined in this case since when $\mathcal{F}=i d$ the walk is transient by Thomassen result. Therefore, by using (8), we get

$$
\mathbb{E}_{o}\left(l_{B(o, R)}\right) \geq \frac{\epsilon}{2}\left(\frac{R}{\alpha}-N_{\alpha}\right) .
$$

By Proposition 2.7 and since strong anchored isoperimetric inequality implies a subexponential volume growth, there exists $c>0$ such that:

$$
\mathbb{E}_{o}\left(l_{B(o, R)}\right) \leq \ln (|B(o, R)|) \leq c R
$$

Choose now $\alpha$ such that $\frac{\epsilon}{2 \alpha}>c$. Gathering (9)and (10), we get:

$$
\frac{\epsilon}{2}\left(\frac{R}{\alpha}-N_{\alpha}\right) \leq c R
$$

Letting $R$ goes to infinity in this last expression, we get a contradiction.

3.3. Random environment on $\mathbb{Z}^{d}$, including supercritical percolation. After a presentation of random environment, we state an anchored isoperimetric inequality for big sets under super-critical exponentially integrable conductances (see below), which leads to occupation time estimate for big sets.

Consider the graph $\mathcal{L}^{d}=\left(\mathbb{Z}^{d}, E_{d}\right)$ where $E_{d}$ contains non-oriented nearestneighbor pairs. We write $x \sim y$ if $\{x, y\} \in E_{d}$. An environment is a random function $\mu^{\omega}: E_{d} \rightarrow\left[0 ;+\infty\left[\right.\right.$. It is implicit in the definition of $E_{d}$ that it is symetric. The value $\mu^{\omega}(x, y)$ is called the conductance of edge $x, y$. To enlighten the notations, we will sometimes write $\mu$ instead of $\mu^{\omega}$ when there is no ambiguity.

Let $\mathbb{Q}$ be a product probability measure on $\left[0 ;+\infty\left[{ }^{E_{d}}\right.\right.$. A walker or an electric current can cross only edges with strictly positive conductances. So we call cluster a connected component of the graph $\left(\mathbb{Z}^{d},\left\{e \in E_{d} ; \omega(e)>0\right\}\right)$ and we use $\mathbb{Q}$ connectedness refering to this graph. In fact $\mathbb{Q}$ induces a Bernoulli percolation $P_{\mathbb{Q}}$ of parameter $q=\mathbb{Q}(\mu(e)>0)$ (here and in the following, $e$ is any edge since $\mathbb{Q}$ is a product measure). We assume $q>p_{c}$ critical parameter of edge percolation on $\mathbb{Z}^{d}$.

Definition 3.2. For $q>p_{c}$, the law $\mathbb{Q}$ is said to be a super-critical exponentially integrable random environment if there exists $\beta>0$ such that

$$
\mathbb{E}_{\mathbb{Q}}(\exp (\beta \mu(e)))<\infty
$$


For quenched results on the random walk, we consider measure $\mathbb{P}_{0}=\mathbb{P}_{0, \mu^{\omega}}(\cdot \mid$ $\mathcal{C}_{0}$ infinite). That is, we start the random walk from the origin 0 of $\mathbb{Z}^{d}, \mu$ induces $m$ and $p(\cdot, \cdot)$ so that we are in the setting defined in Section 1.3, and we assume the cluster $\mathcal{C}_{0}$ of 0 is infinite.

In order to apply our results, we need an anchored isoperimetric inequality with respect to random weight $\mu^{\omega}$. Differents forms of strong isoperimetric inequality have been established by many authors (see [18], [22] [12] and [5]) in the percolation context.

We may only have a control for big sets. The form which seems adapted to our exit time results is the following:

Proposition 3.3. Let $\mathbb{Q}$ be a super-critical exponentially integrable random environment on $\mathbb{Z}^{d}$.

There exist $\beta_{0}(\mathbb{Q})>0$ and a random integer $N_{0}(\omega)$ such that, for all $\mathbb{Q}$-connected sets $A \subset \mathbb{Z}^{d}$ containing 0 ,

$$
|A| \geq N_{0}(\omega) \quad \Longrightarrow \quad \frac{\mu^{\omega}(\partial A)}{m^{\omega}(A)^{1-1 / d}} \geq \beta_{0} .
$$

The details of the proof can be found in [11]. We use standard exponential Bienaymee Tchebytchef inequality and renormalization technique (see [2]).

Remark 3.4. In [6], it is proved that we can build environments where the return probability is greater than $1 / n^{2}$. By our Proposition 3.3, the d-dimensional anchored isoperimetric inequality is satisfied on these environments and so in dimension higher than 4, no one can hope to prove that in this case, the return probability is in $n^{-d / 2}$.

We can now apply result of Theorem 1.4 in the particular case of random walk in random environment with $\mathbb{Q}$ a super-critical exponentially integrable random environment. We get,

Proposition 3.5. There exists constant $C=C(Q, d)$ such that $Q$ a.s. for all environment $\omega, Q$ super-critical exponentially integrable environment:

for any connected subset $B$ which contains the origin and with volume $|B|$ large enough,

$$
\left\{\begin{array}{l}
\mathbb{E}_{0}\left(l_{B}\right) \leq C m^{\omega}(B) \quad \text { in dimension } d \geq 3 \\
\mathbb{E}_{0}\left(\tau_{B}\right) \leq C m^{\omega}(B) \quad \text { for dimension } d=2
\end{array}\right.
$$

For transient case, the proof consists in solving the differential inequation:

$$
\left\{\begin{array}{l}
u(0)=m^{\omega}(B) \\
u^{\prime} \leq-\left(\beta_{0} u^{1-\frac{1}{d}}\right)^{2}, \quad \text { until } \#\{x \in B ; G(0, x) \geq t\} \geq N_{0}(\omega),
\end{array}\right.
$$

satisfied by $u(t)=m^{\omega}(\{x \in B ; G(0, x) \geq t\})$. Then, using Corollary 3.2, you get the expectation of the occupation time. The argument is similarly for $d=2$. The complete proof is in [11].

Percolation is a particular case of $Q$ super-critical exponentially integrable random environment. So, theses results hold for percolation super-critical cluster.

Proposition 3.6. Let $p>p_{c}(d)$ and $d \geq 2$. There exists constant $C=C(p, d)$ such that $Q$ a.s. on the event $\{\# \mathcal{C}=+\infty\}$ : 
for any connected subset $B$ of $\mathcal{C}$ which contains the origin and with volume large enough,

$$
\begin{cases}\mathbb{E}_{0}\left(l_{B}\right) \leq C|B|^{2 / d} & \text { if } d \geq 3 \\ \mathbb{E}_{0}\left(\tau_{B}\right) \leq C|B| & \text { if } d=2\end{cases}
$$

These estimates have the right behaviour, since we retrieve a consequence of results of Barlow. Indeed, in [3], Barlow gives a precise control of the kernel transitions of the random walk. It is proved that:

Theorem 3.7. There exists $\Omega_{1}$ with $Q\left(\Omega_{1}\right)=1$ and random variables $S_{x} ; x \in \mathbb{Z}^{d}$ such that for each $x \in \mathcal{C}$ and for all $\omega \in \Omega_{1}, S_{x}(\omega)<\infty$ and there exist constants $c i=c_{i}(d ; p)>0$ such that for all $x, y \in \mathcal{C}$ and $t \geq 1$ with

$$
k \geq S_{x}(\omega) \vee|x-y|_{1}
$$

the transition density $\mathbb{P}_{x}\left(X_{k}=y\right)$ of $X$ satisfies:

$$
\nu(y) c_{1} k^{-d / 2} e^{-c_{2} \frac{|x-y|_{1}^{2}}{k}} \leq \mathbb{P}_{x}\left(X_{k}=y\right) \leq \nu(y) c_{3} k^{-d / 2} e^{\frac{-c_{4}|x-y|_{1}^{2}}{k}} .
$$

As the expectation of exit time can be expressed with the kernel transitions, these estimates give us a bound of the exit time of the correct order. (see [11] for details)

Acknowledgments: The authors would like to thank Noam Berger, Gady Kozma and Pierre Mathieu for their comments on earlier version of the paper.

\section{REFERENCES}

[1] S. Alexander. and R. Orbach. Density of states on fractals: "fractons". J. Physique (Paris) Lett., 43:625-631, 1982.

[2] P. Antal and A. Pisztora. On the chemical distance for supercritical Bernoulli percolation. Ann. Probab., 24(2):1036-1048, 1996.

[3] M.T. Barlow. Random walks on supercritical percolation clusters. Ann. Probab., 32(4):30243084, 2004.

[4] I. Benjamini, R. Lyons, and O. Schramm. Percolation perturbations in potential theory and random walks. In Random walks and discrete potential theory (Cortona, 1997), Sympos. Math., XXXIX, pages 56-84. Cambridge Univ. Press, Cambridge, 1999.

[5] N. Berger, M. Biskup, C. Hoffman, and G. Kozma G. Anomalous heat-kernel decay for random walk among bounded random conductances. 2007.

[6] O. Boukhadra. Anomalous heat-kernel decay for random walk among polynomial lower tail random conductances. 2008.

[7] D. Chen and Y. Peres. Anchored expansion, percolation and speed. Ann. Probab., 32(4):29782995, 2004. With an appendix by Gábor Pete.

[8] T. Coulhon. Ultracontractivity and Nash type inequalities. J. Funct. Anal., 141(2):510-539, 1996.

[9] T. Coulhon and L. Saloff-Coste. Puissances d'un opérateur régularisant. Ann. Inst. H. Poincaré Probab. Statist., 26(3):419-436, 1990.

[10] P.G. de Gennes. La percolation : un concept unificateur. La Recherche, 7:919-927, 1976.

[11] T. Delmotte and C. Rau. Exit time for anchored expansion. http://arxiv.org/abs/0903.3892, 2008.

[12] P. Gabor. A note on percolation on $\mathbb{Z}^{d}$, isoperimetric profile via exponential cluster repulsion. 2008.

[13] A. Grigor'yan. On the existence of positive fundamental solution of the laplace equation on riemannian manifolds. Mat. Sb. (N.S.), 56(2):349-358, 1987.

[14] A. Grigor'yan. Heat kernel upper bounds on a complete non-compact manifold. Rev. Mat. Iberoamericana, 10(2):395-452, 1994.

[15] G.R. Grimmett. Percolation. 1989. 
[16] R.V.D Hofstad. The incipient infinite cluster for high-dimensional unoriented percolation. Journal of Statistical Physics, 2011.

[17] R. Lyons, B. Morris, and O. Schramm. Ends in uniform spanning forests. Electron. J. Probab., 13:no. 58, 1702-1725, 2008.

[18] P. Mathieu and E. Remy. Isoperimetry and heat kernel decay on percolation clusters. Ann. Probab., 32(1A):100-128, 2004.

[19] B. Morris and Y. Peres. Evolving sets and mixing. In Proceedings of the Thirty-Fifth Annual ACM Symposium on Theory of Computing, pages 279-286 (electronic), New York, 2003. ACM.

[20] J. Nash. Continuity of solutions of parabolic and elliptic equations. Amer. J. Math., 80:931954, 1958.

[21] C. Pittet and L. Saloff-Coste. A survey on the relationships between volume growth, isoperimetry, and the behaviour of simple random walk on cayley graphs, with examples. Preprint, 2001.

[22] C. Rau. Sur le nombre de points visités par une marche aléatoire sur un amas infini de percolation. Bull. Soc. Math. France, 135(1):135-169, 2007.

[23] O. Schramm and Z. Xu. Hyperbolic and parabolic packings. Discrete Comput. Geom., 14(2):123-149, 1995.

[24] Y.G. Sinai. Theory of phase transition: Rigourous results. Int Series in Natural Phil., 108.

[25] C. Thomassen. Isoperimetric inequalities and transient random walks on graphs. Ann. Probab., 20(3):1592-1600, 1992.

[26] N. Th. Varopoulos. Hardy-Littlewood theory for semigroups. J. Funct. Anal., 63(2):240-260, 1985.

[27] B. Virág. Anchored expansion and random walk. Geom. Funct. Anal., 10(6):1588-1605, 2000.

Thierry Delmotte

Université Paul Sabatier

Institut de Mathématiques de Toulouse

route de Narbonne

31400 Toulouse

Thierry.Delmotte@math.ups-tlse.fr

\section{Clément RAU}

Université Paul Sabatier

Institut de Mathématiques de Toulouse

route de Narbonne

31400 Toulouse

rau@math.ups-tlse.fr

$U R L:$ http: //www . math.univ-toulouse.fr/ delmotte

$U R L$ : http://www.math. univ-toulouse.fr/ rau/ 